\title{
A133 EFFECT OF IMMUNOSUPPRESSIVE TREATMENT ON INTERLEUKIN 15 IN MUSCLE TISSUE OF PATIENTS
}

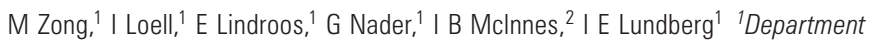
of Medicine, Rheumatology Unit, Karolinska Institutet/Karolinska University Hospital, Solna, Stockholm, Sweden; ${ }^{2}$ Centre for Rheumatic Diseases, University of Glasgow, Glasgow Royal Infirmary, Glasgow, UK

10.1136/ard.2010.129643j

Background Polymyositis and dermatomyositis are chronic inflammatory diseases of primary affecting skeletal muscle where the infiltrates are composed of $T$ cells and where CD8 $\mathrm{T}$ cells are often found. The molecular mechanisms that promote $T$ cell proliferation and cause muscle weakness are still unknown. In this context, interleukin (IL)15 is of potential interest as this cytokine could induce proliferation of CD8 $\mathrm{T}$ cells and could have a role in muscle fibre regeneration.

Objectives To investigate the expression of IL15 and IL15 receptor $\alpha$ (IL15R $\alpha$ ) in muscle biopsies from patients with polymyositis or dermatomyositis before and after conventional immunosuppressive treatment.

Methods Muscle biopsies from 17 patients with polymyositis or dermatomyositis before and after $4-16$ months with conventional immunosuppressive treatment and 7 healthy individuals were investigated by immunohistochemistry using antibododies against IL15 and IL15R $\alpha$. Quantification was performed by computerised image analysis. The number of T lymphocytes and macrophages in muscle tissue was also quantified by the same method. The localisation of expression of IL15 was determined by double staining using immunofluorescence. The functional index was used to assess muscle function.

Results The expression of IL15 was observed predominantly in mononuclear inflammatory cells of muscle tissue from patients, while IL15R $\alpha$ was localised to mononuclear inflammatory cells, capillaries and large vessels. Higher expression of both IL15 and IL15R $\alpha$ in mononuclear inflammatory cells 
was found in myositis patients compared with healthy individuals, but IL15R $\alpha$ expression in capillaries and large vessels did not show a significant difference between patients and controls. There was no difference between polymyositis and dermatomyositis. Double immunofluorescence demonstrated a predominant expression of IL15 in macrophages marked by CD163. After conventional immunosuppressive treatment, significantly reduced expression of IL15 and IL15R $\alpha$ in mononuclear inflammatory cells was detected in the whole group, but 8 patients still had significantly higher IL15 expression than controls. Following the same treatment, no significant changes were observed in muscle tissue regarding $\mathrm{T}$ lymphocytes, CD68-positive macrophages or CD163-positive macrophages. Significantly improved muscle function was found after treatment, but only one patient had recovered muscle function. No correlation was demonstrated between the changes in IL15 and the functional index.

Conclusions In patients with myositis the expression of IL15 and IL15R $\alpha$ in mononuclear inflammatory cells in muscle tissue was significantly higher than in healthy individuals. IL15 was localised to macrophages. After conventional immunosuppressive treatment, both the expression of IL15 and IL15R $\alpha$ in mononuclear inflammatory cells was significantly reduced. However, eight patients who had persisting impaired muscle performance after treatment, still had a higher expression of IL15 than controls, which could indicate a role for IL15 in the pathogenesis of these diseases and that IL15 might be a therapeutic target in a subset of myositis patients. 